\title{
Circulating Neutrophils of Nonalcoholic Steatohepatitis Patients Show an Activated Phenotype and Suppress $T$ Lymphocytes Activity
}

\author{
Laura Antonucci $\mathbb{D}^{1}{ }^{1}$ Cristiana Porcu, ${ }^{1}$ Eleonora Timperi, ${ }^{2}$ Silvano Junior Santini, ${ }^{3}$ \\ Gino Iannucci, ${ }^{2}$ and Clara Balsano ${ }^{1,1,3}$ \\ ${ }^{1}$ Francesco Balsano Foundation, Rome, Italy \\ ${ }^{2}$ Dipartimento di Medicina Interna e Specialità Mediche, Sapienza Università di Roma, Rome, Italy \\ ${ }^{3}$ Department of Life, Health and Environment Sciences, University of L'Aquila, L'Aquila, Italy \\ Correspondence should be addressed to Clara Balsano; clara.balsano@cc.univaq.it \\ Laura Antonucci and Cristiana Porcu contributed equally to this work.
}

Received 18 February 2020; Revised 24 April 2020; Accepted 2 June 2020; Published 30 June 2020

Academic Editor: Enrique Ortega

Copyright (c) 2020 Laura Antonucci et al. This is an open access article distributed under the Creative Commons Attribution License, which permits unrestricted use, distribution, and reproduction in any medium, provided the original work is properly cited.

\begin{abstract}
Neutrophils or PolyMorphonuclear Neutrophils (PMNs) are key effector cells of the innate immune system and thanks to their remarkable plasticity, establish a cross talk with $\mathrm{T}$ cells modulating their survival and effector functions. During Nonalcoholic Steatohepatitis (NASH), the advanced form of hepatic steatosis or NAFL, PMNs infiltrate liver tissue, becoming a histological feature of NASH. Our aim was to evaluate the frequency of PMNs in NAFL and NASH patients in order to understand how they modulate the activity of circulating $\mathrm{CD}^{+}$and $\mathrm{CD}^{+} \mathrm{T}$ cells. In our cohort of patients, NASH patients displayed a higher frequency of circulating PMNs that was strongly correlated to liver enzymes, grade of steatosis, inflammation and fibrosis, the hepatocellular ballooning, and NAFLD Activity Score (NAS). Furthermore, even if ex vivo, in both groups of patients, PMNs shared the same phenotype of resting cells, after 24 hours of coculture with autologous CD4+ and CD8+ T cells, PMNs of NASH patients acquired a more active phenotype, becoming able to strongly inhibit proliferation and activation of $\mathrm{CD}^{+}$and $\mathrm{CD}^{+}{ }^{+} \mathrm{T}$ cells. The higher ability of PMNs of NASH patients in suppressing $\mathrm{CD} 4^{+}$and $\mathrm{CD} 8^{+} \mathrm{T}$ cells, over time, might contribute in reducing the immunological defense of liver tissue against damages thus taking part in the progression of the NAFL disease toward NASH.
\end{abstract}

\section{Introduction}

Nonalcoholic fatty liver disease (NAFLD) is a very common chronic disease, ranging from simple hepatic steatosis (NAFL), due to an excessive fat deposition in the hepatocytes without any sign of inflammation or necrosis, to nonalcoholic steatohepatitis (NASH), featured by steatosis and hepatic inflammation [1-3]. NASH can lead to advanced fibrosis and cirrhosis, thereby increasing the risk of developing hepatocellular carcinoma (HCC) [4].

Despite NAFL affects around one-third of the Western world with an incidence that continues to grow, no pharmacological drugs have been already approved for the treatment of the disease. Currently, only lifestyle change (diet and physical activity) is suggested to these patients $[5,6]$.

During NASH disease, stressed or dying hepatocytes release intracellular molecules called damage-associated molecular patterns (DAMPs), such as high mobility group box 1 (HMGB1), that induce immune cells to initiate a homeostatic wound-healing response to repair liver injury [7]. Accordingly, DAMPs stimulate Kupffer cells (KCs) to produce guidance signals such as macrophage inflammatory protein-2 (MIP-2), interleukin-1 $\beta$ (IL-1 $\beta$ ), tumor necrosis factor- $\alpha$ (TNF- $\alpha$ ), and interleukin-8 (IL-8) to recruit polymorphonuclear neutrophils (PMNs) within the portal tract $[8,9]$. Amongst the others, IL- 8 represents the most potent 
PMN chemo-attractants in inflammation, which, through binding with two different chemokine receptors $\mathrm{C}-\mathrm{X}-\mathrm{C}$ chemokine receptor type 1 (CXCR-1) and C-X-C chemokine receptor type 2 (CXCR-2), induces specific intracellular signaling cascades for a rapid PMNs recruitment $[10,11]$.

PMNs accumulation in the liver tissue is one of the main features of NASH, contributing to liver damage and exacerbating inflammatory state [12]. PMNs represent the most abundant circulating white blood cells in humans [13]. Their mobilization out of blood vessels into injured tissue is a highly regulated process that depends on sequential interactions between adhesion molecules present in the environment, such as selectins [L-selectin (CD62L), P-selectin (CD62P), Eselectin (CD62E)], integrins [integrin $\alpha \mathrm{M}$ (CD11b), $\beta 2$ integrin $(\alpha \mathrm{L} \beta 2)$ ], and chemokines [14]. In particular, CD62L is expressed on PMNs surface and has an important role in the initial rolling and attachment of PMNs to the endothelium [15]. This is followed by tight adhesion and transendothelial migration mediated by integrins, such as CD11b. Therefore, the activation of PMNs is associated to the shedding of CD62L and the upregulation of CD11b. Once captured and activated, adherent PMNs migrate through the vessel wall to infiltrate the tissue [16].

PMNs functions are mediated by the expression levels of myeloperoxidase (MPO), arginase-1 (ARG1), and reactive oxygen species (ROS), which reflect PMN activity, and their involvement in inducing cellular injury and fibrosis [8, 17]. Activated PMNs promote the recruitment of lymphocytes by the release of mediators of inflammation and chemokines [18-20]. As a result, resident and infiltrated $\mathrm{T}$ cells release cytokines that, acting on the endothelial cells of the liver sinusoids, lead to the production of IL- 8 for a further recruitment of PMNs. This interplay between PMNs and T cells intensifies the state of inflammation and creates a link between the adaptive and the innate immune system [20]. PMNs establish a close interaction with $\mathrm{T}$ cells to promote activation or suppression of their effector functions, influencing, in this way, the state of different pathologies, such as infections, sepsis, and cancer [21-24]. For example, in HIV-1 infected patients, PMNs suppress $\mathrm{T}$ cell activity lading to an acceleration of the disease [22]. PMNs have also antitumor and protumor activities. Accordingly, during the early phase of lung tumor growth, PMNs tend to inhibit primary tumor growth by recruiting and activating $\mathrm{T}$ cells [25-27]. On the contrary, in the late stage of cancer, PMNs show an immunosuppressive phenotype that suppresses antitumor T-cell responses and promotes immune evasion [28]. Thus, as extensively reported in the literature, PMNs can positively or negatively modulate T-cell functions. PMNs can activate a variety of T-cell subsets, while, the inhibition of T-cells by immunosuppression of PMNs occurs through the release of ARG1, ROS, and proteases, or via PD-L1-dependent interaction [20, 22].

The modulation of T cell responses by PMNs has not yet been studied in NAFL and NASH patients. This interplay is extremely important since it might influence the state and progression of NAFLD disease. We analyzed the frequency and phenotype of PMNs in healthy donors (HD), NAFL, and NASH patients wondering how PMNs may drive and modulate the activity of $\mathrm{T}$ cells in this disease.

\section{Material and Method}

2.1. Patients and Biochemical Parameters. Observational study on 10 healthy donors and 30 consecutive patients with a diagnosis of NAFL/NASH performed by ultrasound and by excluding the following criteria: no history of current or past excessive alcohol drinking as defined by an average daily consumption of alcohol $<30 \mathrm{~g} /$ day in men and $<20 \mathrm{~g} /$ day in women; negative tests for the presence of hepatitis $B$ surface antigen and antibody to hepatitis $C$ virus; absence of history and findings consistent with cirrhosis and other chronic liver diseases [29]. Human studies were performed in accordance with the ethical guidelines of the 1975 Declaration of Helsinki and approved by the Ethical Committee of the University of L'Aquila, L'Aquila, Italy (Prot. No. 31869). Informed consent was obtained from all enrolled patients.

All subjects, at the time of enrolment, had a complete work-up including a clinical examination, anthropometric measurements, laboratory tests, and a liver ultrasonography scan. Blood sampling was performed in the morning after an overnight fast. Patient characteristics are listed in Table 1.

Study population underwent fasting blood sampling to assess blood glucose (FBG), total cholesterol, HDL-cholesterol, triglycerides, aspartate aminotransferase (AST), alanine aminotransferase (ALT), $\gamma$-glutamyl transpeptidase $(\gamma$-GT), alkaline phosphatase, nitrogen, and creatinine by standard laboratory methods. Low-density lipoprotein (LDL) cholesterol value was obtained using the Friedwald formula. The homeostasis model assessment of insulin resistance (HOMA-IR) was calculated as previously described [30]. Peripheral blood neutrophilto-lymphocyte ratio (NLR) was calculated dividing the number of neutrophils by the number of lymphocytes in peripheral blood sample.

Evaluation Liver ultrasonography scanning was performed to assess the degree of steatosis. All ultrasonography were performed by the same operator who was unaware of the aims of the study and blinded to laboratory values using an EsaoteMedica apparatus equipped with a convex 3,5 $\mathrm{MHz}$ probe.

Steatosis was graded according to Matsuda and DeFronzo [31] on the basis of abnormally intense, high-level echoes arising from the hepatic parenchyma, liver-kidney difference in echo amplitude, echo penetration into the deep portion of the liver, and clarity of liver blood vessel structure.

2.2. Liver Histology. Only in NASH group patients it was possible to carry out biopsy, as indicated in the American Association for the Study of Liver Diseases (AASLD) guidelines [32-34].

The histological diagnosis was established using Hematoxylin \& eosin ( $\mathrm{H} \& \mathrm{E})$ and Masson trichrome stains of formalin-fixed paraffin-embedded liver tissue. All biopsies were evaluated by a pathologist blinded to patient data according to the NAFLD Activity Score- (NAS-) scoring system described by Kleiner et al. [35] to detect the number of patients who met the histological criteria for nonalcoholic steatohepatitis (NASH). The histological features of liver tissues, commonly described in NASH, including steatosis, inflammation (portal and lobular), hepatocyte ballooning, and fibrosis were scored as summarized in Table 2. 
TABLE 1: Anthropometric and biochemical parameters in healthy donors, NAFL, and NASH patients.

\begin{tabular}{|c|c|c|c|c|c|}
\hline Parameters & $\begin{array}{l}\text { Healthy donors } \\
\text { (10) }\end{array}$ & NAFL (10) & NASH (20) & $\begin{array}{l}P \text { value (NASH vs. healthy } \\
\text { donors) }\end{array}$ & $\begin{array}{c}P \text { value (NASH vs. } \\
\text { NAFL) }\end{array}$ \\
\hline Age (years) & $54 \pm 6.9$ & $57.6 \pm 7.2$ & $56.3 \pm 11.2$ & 0.1682 & 0.8655 \\
\hline $\operatorname{Sex}(F / M)$ & $5 / 5$ & $4 / 6$ & $12 / 8$ & & \\
\hline Weight (kg) & $68.75 \pm 5.3$ & $69.4 \pm 8.3$ & $88.7 \pm 17.3$ & $<0.0001$ & $<0.0001$ \\
\hline BMI $\left(\mathrm{kg} / \mathrm{m}^{2}\right)$ & $22.4 \pm 1.68$ & $23.0 \pm 1.7$ & $32.4 \pm 4.7$ & $<0.0001$ & $<0.0001$ \\
\hline AST (UI/mL) & $11.2 \pm 5.5$ & $20.4 \pm 6.5$ & $30.7 \pm 11.3$ & 0.0046 & 0.0007 \\
\hline $\operatorname{ALT}(\mathrm{UI} / \mathrm{mL})$ & $12.1 \pm 4.04$ & $26.1 \pm 5.53$ & $62.8 \pm 30.2$ & 0.0006 & 0.0005 \\
\hline GGT (UI/mL) & $18.52 \pm 5.58$ & $23.9 \pm 7.39$ & $61.2 \pm 20.2$ & $<0.0001$ & $<0.0001$ \\
\hline Total cholesterol (mg/dL) & $161 \pm 15.5$ & $172.9 \pm 24.1$ & $212 \pm 25.9$ & $<0.0001$ & $<0.0001$ \\
\hline LDL cholesterol (mg/dL) & $100.6 \pm 20.9$ & $109.7 \pm 33.8$ & $125 \pm 19.5$ & 0.0432 & 0.0339 \\
\hline HDL cholesterol (mg/dL) & $48.0 \pm 8.1$ & $47.6 \pm 7.3$ & $46.8 \pm 9.9$ & 0.7802 & 0.1406 \\
\hline Triglycerides (mg/dL) & $100.8 \pm 25.7$ & $101.8 \pm 33.2$ & $117.3 \pm 67.4$ & 0.0169 & 0.0004 \\
\hline Glucose (mg/dL) & $85.4 \pm 10.0$ & $92.2 \pm 7.6$ & $99.8 \pm 16.5$ & 0.0264 & 0.0173 \\
\hline Insulin (UI/mL) & $6.4 \pm 1.7$ & $8.45 \pm 4.2$ & $23.1 \pm 6.2$ & $<0.0001$ & $<0.0001$ \\
\hline $\begin{array}{l}\text { Alkaline phosphatase } \\
\text { (UI/L) }\end{array}$ & $93 \pm 45.2$ & $112.9 \pm 40.4$ & $125.8 \pm 47$ & 0.3044 & 0.0923 \\
\hline Total bilirubin (mg/dL) & $0.6 \pm 0.2$ & $0.7 \pm 0.2$ & $0.7 \pm 0.3$ & 0.1883 & 0.9803 \\
\hline Direct bilirubin (mg/dL) & $0.2 \pm 0.01$ & $0.2 \pm 0.04$ & $0.2 \pm 0.1$ & 0.0524 & 0.5823 \\
\hline HOMA-IR & $1.08 \pm 0.22$ & $1.08 \pm 1.1$ & $5.6 \pm 2.2$ & $<0.0001$ & $<0.0001$ \\
\hline SBP (mmHg) & $133 \pm 9.8$ & $135 \pm 8.9$ & $140 \pm 11.7$ & 0.2268 & 0.0684 \\
\hline DBP (mmHg) & $70.4 \pm 2.9$ & $73.4 \pm 5.2$ & $82.2 \pm 8.8$ & 0.0072 & $<0.0001$ \\
\hline Leucocyte $\left(10^{3} / \mu \mathrm{L}\right)$ & $4.66 \pm 0.9$ & $5.12 \pm 0.8$ & $6.6 \pm 2.0$ & 0.0359 & 0.0586 \\
\hline Neutrophil $\left(10^{3} / \mu \mathrm{L}\right)$ & $1.86 \pm 0.13$ & $1.9 \pm 0.4$ & $4.1 \pm 1.3$ & 0.0002 & 0.0003 \\
\hline Lymphocyte $\left(10^{3} / \mu \mathrm{L}\right)$ & $1.65 \pm 0.31$ & $1.6 \pm 0.4$ & $1.9 \pm 0.6$ & 0.6981 & 0.3611 \\
\hline NLR & $1.1 \pm 0.27$ & $1.3 \pm 0.5$ & $2.4 \pm 0.8$ & 0.0021 & 0.0093 \\
\hline
\end{tabular}

BMI: body mass index; AST: aspartate aminotransferase; ALT: alanine aminotransferase; GGT: Gamma glutamil transferase; HOMA-IR: homeostasis model assessment of insulin resistance; LDL: Low Density Lipoprotein; HDL: High Density Lipoprotein; SBP: systolic blood pressure; DBP: systolic blood pressure; NLR: Neutrophil-to-lymphocyte ratio. Normally distributed date described as mean, standard deviations (SDs), $P$ value, ${ }^{*} P<0.05,{ }^{* *} P<0.01,{ }^{* * *} P<0.001$, ${ }^{* * * *} P<0.0001$, and unpaired Mann-Whitney $U$ test.

2.3. Enzyme-Linked Immunosorbent Assay (ELISA). IL-8 (Diaclone, Cat\# 855.080.005, RRID:AB_596520, France), HMGB1 (Tecan Trading AG, Switzerland), myeloperoxidase (MPO) (Enzo Life Sciences, Inc.), and arginase 1 (ARG1) (Elabscince, USA) concentrations were measured in the serum of NAFL and NASH patients, with specific immunoassay kits, according to the manufacturer's instructions. Absorbance was read at $450 \mathrm{~nm}$ with a photometer (Multiskan, flow cytometry, TermoFisher). All samples were analyzed in duplicate in the same run.

2.4. Isolation of PBMCs and PMNs. PMNs and peripheral blood mononuclear cells (PBMCs) were isolated from heparinized venous blood from NAFL and NASH patients. Cells were isolated by density gradient centrifugation by Lympholyte (Cedarlane, Burlington, ON, Canada). The low-density fraction of PBMCs was removed and, after wash, collected in complete RPMI 1640 medium containing 10\% FBS (HyClone GE Healthcare Life Sciences), 2 mM L-6-glutamine (SigmaAldrich), penicillin/streptomycin (EuroClone).
PMNs obtained from high-density fraction by sedimentation (30 minutes) of the red blood cells with 3\% Dextran/HBSS without calcium and magnesium (Sigma-Aldrich) followed by lysis of the erythrocytes by the usage of Red Blood Cell Lysis Solution (RBC 10x, MiltenyBiotec). To check the purity of isolated PMNs, CD11b (Thermo Fisher Scientific Cat\# 14-011282, RRID:AB_467108) and CD16 (Thermo Fisher Scientific Cat\# 46-0168-41, RRID:AB_1834390) markers are analyzed by flow cytometry (FC). The purity of PMNs exceeded the $90 \%$. PMNs viability was greater than $98 \%$, as determined by Trypan blue (Sigma-Aldrich) exclusion. PMNs were suspended in HBSS (without $\mathrm{Ca}^{2+} / \mathrm{Mg}^{2+}$ ) and placed on ice until use.

2.5. Flow-Cytometry Analysis. The list of Antibodies (Abs) used is available on Table 3.

Flow cytometric analysis was performed on PMNs and PBMCs of healthy donors, NAFL, and NASH patients. Dead cells in all samples were excluded using Fixable Viability Dye eFluor 780 (eBioscience) and Zombie Aqua Fixable Viability Kit (Biolegend). Surface staining was performed by 
TABLE 2: Histological characteristics of NASH liver tissues.

\begin{tabular}{lc}
\hline Score & NASH patients $n=20$ \\
\hline Steatosis & \\
0 & $2(10 \%)$ \\
1 & $6(30 \%)$ \\
2 & $12(60 \%)$ \\
3 & \\
Inflammation & $2(10 \%)$ \\
0 & $5(25 \%)$ \\
1 & $13(65 \%)$ \\
2 & \\
Ballooning & $1(5 \%)$ \\
0 & $7(35 \%)$ \\
1 & $12(60 \%)$ \\
2 & \\
NAS & $10(50 \%)$ \\
5 & $4(20 \%)$ \\
6 & $6(30 \%)$ \\
7 & \\
Fibrosis & $6(30 \%)$ \\
0 & $11(55 \%)$ \\
1 & $3(15 \%)$ \\
2 & 0 \\
3 & \\
\hline Aco & \\
& \\
&
\end{tabular}

According to semiquantitatively score described by Gunn and Shiffman [34], the presence of NASH was defined according to the NAFLD activity score (NAS) and the histological features of liver biopsies scored as reported above. The data are showed as prevalence case $\mathrm{N}(\%)$.

incubating the cells with selected $\mathrm{Abs}$ at $4^{\circ} \mathrm{C}$ for $20 \mathrm{~min}$ in PBS 2\% FBS. BD LSR Fortessa cell analyzer (BD Biosciences) was used to acquire the samples and analyzed with FlowJo software, version 10.0.8r1 (Treestar) according to the guidelines for the use of flow cytometry and cell sorting in immunological studies [36]. FMO (fluorescence minus one) staining control was stained with all the antibodies used in the experiment except one which was used as control.

2.6. PBMCs Functional Assays. PBMCs were resuspended in PBS (CambrexBioScience) and labeled with $1 \mu \mathrm{M}$ of carboxyfluoresceindiacetate, succinimidyl ester (CFSE) (Life Technologies, Thermo Fisher Scientific, Grand Island, NY, USA) for $20 \mathrm{~min}$ at $37^{\circ} \mathrm{C}$. After incubation, the cells were washed with complete RPMI 1640 to block the excess of CFSE. Then, PBMCs were cultured in flat-bottomed 96-well plates (Corning B.V. Life Sciences, Amsterdam, Netherlands) in complete RPMI medium and were stimulated with Dynabeads Human T-Activator CD3/CD28 (Thermo-Fisher Scientific) at a ratio of $1: 1$. PMNs were added to autologous PBMCs, and after 4 days of culture, PBMC proliferation and CFSE dilution were analyzed by flow cytomety on gated $\mathrm{CD}^{+}$and $\mathrm{CD}^{+} \mathrm{T}$ lymphocytes.

2.7. Analysis of Markers of PMN Activation. CD62L and CD11b, markers of activation of PMNs, were analyzed in freshly PMNs isolated by pheripharial blood and after 1 day of coculture with autologous PBMCs to evaluate their activation status. After isolation ex vivo, PMNs of NAFL and NASH patients were added to autologous PBMCs at ratio $2: 1$. After $24 \mathrm{~h}$, the surface staining was performed by incubating the cells with selected $\mathrm{Abs}$ at $4^{\circ} \mathrm{C}$ for $20 \mathrm{~min}$ in PBS 2\% FBS. Samples were acquired on the BD LSR Fortessa cell analyzer and analyzed with FlowJo software. CD62 L and CD11b antibodies used for flow cytometry analysis were reported in Table 3. FMO (fluorescence minus one) staining control was stained with all the antibodies used in the experiment except one which was used as control. Flow cytometry compensation beads (Thermo-fisher) were used as positive control of CD62L and CD11B staining on fresh PMNs. PMNs stimulated with Lipopolysaccharide (LPS) $(1 \mathrm{ug} / \mathrm{mL}$, from Escherichia coli O111:B4) were used as a positive control for the detection of CD62L and CD11b expression in coculture experiments.

2.8. Analysis of the Viability of PMNs and PBMCs. The apoptosis of PBMCs and PMNs was assessed by labeling cells with Fixable Viability Dye and Annexin V (Apoptosis detection iteBioscience). The cells were stained with Fixable Viability Dye for $30 \mathrm{~min}$ at room temperature (RT) and washed twice with PBS. Cells were labelled with CD45, CD3, CD4, and CD8 antibodies for the identification of $\mathrm{CD} 4+/ \mathrm{CD} 8+\mathrm{T}$ cells, while CD16, CD62L, and CD11b for detecting PMNs. All antibodies used for flow cytometry analysis were summarized in Table 3. Subsequently, 1/500 Annexin-V diluted was added in appropriated binding buffer (provided by eBioscience kit) for 25 minutes at RT. After incubation, cells were washed in PBS and analyzed by flow cytometry.

2.9. Measurement of Intracellular ROS. $2^{\prime}, 7^{\prime}$-dichlorodihydrofluorescein diacetate (H2DCF-DA) fluorescent probe (Thermo Fischer Scientific) was used to identify the intracellular production of ROS in PMNs and PBMCs cell populations. The cells were stained with Fixable Viability Dye for 30 min at RT then PMNs and PBMCs were labeled with cocktail containing H2DCF-DA probe $(10 \mu \mathrm{M})$ and the following antibodies: CD45, CD3, CD4, and CD8 antibodies for the identification of CD4+/CD8+ T cells, while CD16, CD62L, and CD11b for detecting PMNs. PMNs stimulated with Lipopolysaccharide (LPS) $(1 \mu \mathrm{g} / \mathrm{mL}$, from Escherichia coli O111:B4) were used as positive control for ROS detection.

2.10. Statistical Analysis. 2-tailed paired/unpaired Student $t$ test was used to analyze in vitro data. 2-tailed MannWhitney $U$-test was applied to compare groups of ex vivo samples. Correlations were calculated by using Spearman analysis. In all tests, differences were considered statistically significant when $P$ value was less than 0.05 . Statistical analysis was performed with Prism software (version 6; GraphPad Software, La Jolla, California).

\section{Results}

3.1. Frequency of PMNs and Inflammatory Mediators in NASH Patients. We performed an observational study on 10 healthy donors, 10 NAFL, and 20 NASH patients, enrolled 
TABLE 3: Fluorochrome-antibody combinations used for flow cytometry.

\begin{tabular}{lcclcc}
\hline Human & Fluorochrome & Clone & Company & Dilution in $100 \mu \mathrm{L}$ staining volume & Catalogue number and RRID \\
\hline CD62L & FITC & DREG-56 & BioLegend & $1: 100(1 \mu \mathrm{L})$ & Cat\# 304803, RRID:AB_314463 \\
CD16 & PE & 3G8 & BioLegend & $1: 100(1 \mu \mathrm{L})$ & Cat\# 980102, RRID:AB_2616616 \\
CD56 & PE/dazzle & HCD56 & BioLegend & $1: 40(2.5 \mu \mathrm{L})$ & Cat\# 362543, RRID:AB_2565921 \\
CD14 & PerCP/Cy5.5 & HCD14 & BioLegend & $1: 50(2 \mu \mathrm{L})$ & Cat\# 325621, RRID:AB_893252 \\
CD3 & PerCP/Cy5.5 & OKT3 & BioLegend & $1: 50(2 \mu \mathrm{L})$ & Cat\# 344807, RRID:AB_10641704 \\
CD11b & PE/Cy7 & ICRF44 & BioLegend & $1: 40(2.5 \mu \mathrm{L})$ & Cat\# 301321, RRID:AB_830643 \\
CD45 & APC & 2D1 & BioLegend & $1: 100(1 \mu \mathrm{L})$ & Cat\# 368511, RRID:AB_2566371 \\
CD3 & BrilliantViolet605 & OKT3 & BioLegend & $1: 70(1.4 \mu \mathrm{L})$ & Cat\# 317331, RRID:AB_2561376 \\
CD4 & BrilliantViolet711 & RPA-T4 & BioLegend & $1: 70(1.4 \mu \mathrm{L})$ & Cat\# 300536, RRID:AB_2632791 \\
HLADR & BrilliantViolet711 & L243 & BioLegend & $1: 70(1.4 \mu \mathrm{L})$ & Cat\# 301045, RRID:AB_11219195 \\
CD19 & BrilliantViolet785 & HIB19 & BioLegend & $1: 70(1.4 \mu \mathrm{L})$ & Cat\# 302239, RRID:AB_11218596 \\
CD8 & Brilliant Violet785 & RPA-T8 & BioLegend & $1: 70(1.4 \mu \mathrm{L})$ & Cat\# 301045, RRID:AB_11219195 \\
\hline
\end{tabular}

consecutively by Predictive Medicine Center and Transfusion Center, Policlinico Umberto I-Hospitals-Rome Italy, respectively. PMN profile was examined in the peripheral blood (PB) of healthy donors, NAFL, and NASH patients; the gating strategy has been illustrated in Figure 1(a). We characterized PMNs based on CD16, CD62L, and CD11b expression by flow cytometric analysis. Monocytes and natural killer (NK) cells were excluded based on CD14 and HLADR positivity. No differences were observed in the percentage of classical, intermediate and nonclassical monocytes, and natural killer in the peripheral blood of healthy donors, NAFL, and NASH patients (Supplementary Figure S1). We estimated the percentage of PMNs, identified as SSC High $\mathrm{CD} 16^{\text {High }} \mathrm{CD} 62 \mathrm{~L}^{\text {High }} \mathrm{CD} 11 \mathrm{~b}^{\text {High }}$, in $\mathrm{PB}$ of healthy donors, NAFL, and NASH patients.

We observed that the percentage of PMNs was comparable in NAFL patients and healthy donors, while it was significantly higher in NASH patients compared to healthy donors and NAFL patients (Figure 1(b)). IL-8, MPO, ARG1, and HMGB1 were analyzed in the serum of healthy donors and patients. Notably, all these proinflammatory factors were significantly increased in NASH compared to NAFL patients, while no difference was found between healthy donors and NAFL subjects (Figures 1(c)-1(f)).

3.2. PMN Frequency Directly Correlate to the Severity of the Liver Disease in NASH Patients. To evaluate the clinical implication of PMNs in the pathogenesis of NAFL/NASH liver disease, we correlated the frequency of PMNs, calculated by flow cytometry, with the biochemical parameters of patients. Notably, in NASH patients, we found a significantly positive correlation between $\mathrm{PMN}$ frequency and liver enzymes: AST $\left(r=0.5036 ;{ }^{*} P<0.05\right)$, ALT $\left(r=0.6682 ;{ }^{* *} P\right.$ $<0.01)$, and GGT $\left(r=0.7761 ;{ }^{* * *} P<0.0001\right)$ (Figure $2(\mathrm{a})$ ). No correlations were observed between PMN frequency and alkaline phosphatase, total, and direct bilirubin (Supplementary Figure S2).

After that, in the liver tissue of NASH patients, the possible association between the percentage of PMNs and the severity of the liver damage was evaluated. We look at the grade of steatosis, inflammation and fibrosis, the hepatocellular ballooning, and NAFLD Activity Score (NAS) (Figures 2(b)-2(f), respectively). A strong association between the percentage of PMNs and the severity of liver damage was highlighted (Figures 2(b)-2(f)).

\subsection{Ex Vivo PMNs Resting Phenotype in NAFL and NASH} Patients. We evaluated CD62L and CD11b expression on circulating PMNs purified by healthy donors and NAFL and NASH patients. Ex vivo PMNs of healthy donors, NAFL, and NASH patients showed a resting cell phenotype: $\mathrm{CD} 16^{\text {High }} \mathrm{CD} 62 \mathrm{~L}^{\text {High }} \mathrm{CD} 11 \mathrm{~b}^{\text {High }}$ (Figure $3(\mathrm{a})$ ). Notably, PMNs of NASH patients showed a significant increase of baseline expression of CD62L respect to healthy donors and NAFL patients (Figure 3(a)). On the contrary, no significant difference in the expression of the CD11b was observed in our cohort of healthy donors and patients. CD62L and CD11b expression was estimated by Mean Fluorescence Intensity (MFI).

3.4. Strong Activation State and Prolonged Survival of PMNs in NASH Patients. After 24 hours of coculture with autologous PBMCs, we looked at the expression levels of CD62L and CD11b in PMNs of our cohort of patients (Figure 3(b)). Notably, PMNs of NASH patients displayed a significant decrease in CD62L expression levels and a significant increase in CD11b respect to healthy donors and NAFL patients (Figure 3(b), representative flow cytometry gating strategy showed in Supplementary Figure S3).

PMNs from NASH patients showed, at baseline, high levels of ROS production (Figure 4(a)). Comparing foldchange in ROS production, we highlighted a significant increase in ROS production in PMNs+PBMCs coculture (Supplementary Figure S4). PMNs of NASH patients showed a significant increase of cell survival (\%Annexin V-/Live Dead-) respect to PMNs of HD and NAFL patients, regardless of the presence of PBMCs (Figure 4(b)). Accordingly, a significant decrease of cell death (\%Annexin V+/Live Dead-) in PMN + PBMC of NASH patients respect to PMNs was observed (Figure 4(b)). 

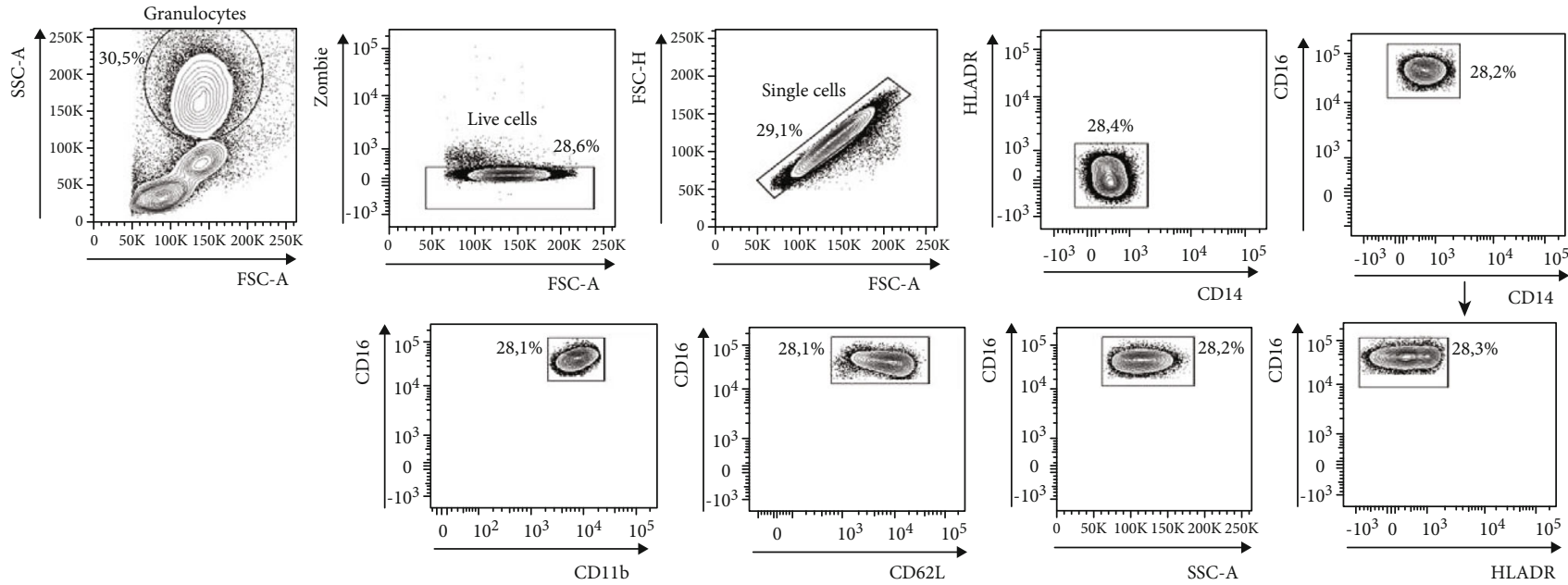

(a)

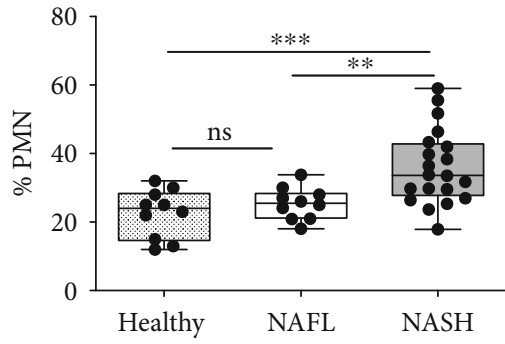

(b)

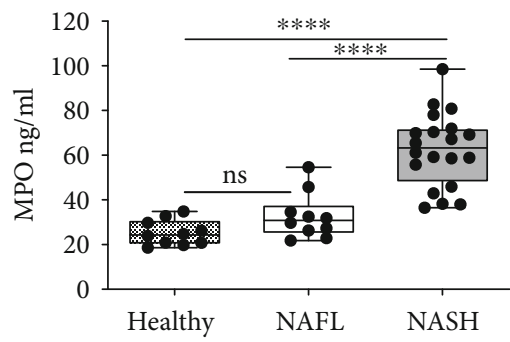

(d)

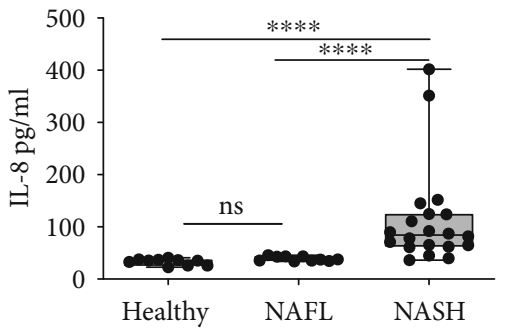

(c)

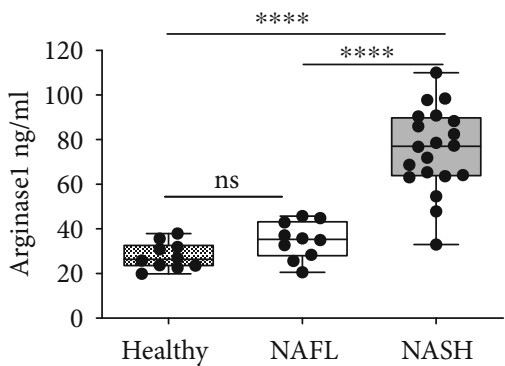

(e)

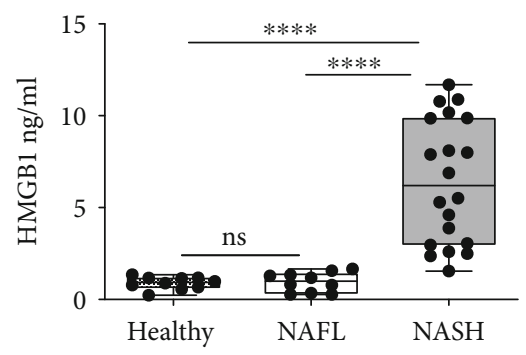

(f)

FIGURE 1: Frequency of PMNs and mediators of inflammation in Healthy Donors, NAFL, and NASH patients. (a) Representative gating strategy for the flow cytometric analysis of PMNs identification from peripharial blood. PMNs are identified as SSChigh CD16 high $\mathrm{CD} 62 \mathrm{~L}^{\text {high }} \mathrm{CD} 11 \mathrm{~b}^{\text {high }}$. (b) Frequency was calculated by flow cytometry analysis as the percentage of CD16 ${ }^{\text {high }} \mathrm{CD} 62 \mathrm{~L}^{\text {high }} \mathrm{CD} 11 \mathrm{~b}^{\text {high }}$ of PMNs in healthy donors $(n=10)$, NAFL $(n=10)$, and NASH patients $(n=20)$. Each dot in the dot-plot represents one subject/experiment. (c-f) Serum concentration of IL-8 pg/mL (Healthy $n=10$, NAFL $n=10$, and NASH $n=20$ ) (c), MPO ng/mL (Healthy $n=10$, NAFL $n=10$, and NASH $n=20)(\mathrm{d})$, ARG1 $\mathrm{ng} / \mathrm{mL}$ (Healthy $n=10$, NAFL $n=10$, and NASH $n=20)(\mathrm{e}$ ), and HMGB1 ng/mL (Healthy $n=10$, NAFL $n=10$, and NASH $n=20$ ) (f) in healthy donors, NAFL, and NASH patients were quantified by ELISA analysis. Each dot in the graph represents a single subject. Each dot in the dot-plot represents one subject/experiment. Each subject has been studied in an independent experiment. Plots are representative for three independent experiment with one sample per experiment. MannWhitney unpaired test, 2 -tailed. $P$ value, ${ }^{*} P<0.05,{ }^{* *} P<0.01,{ }^{* * *} P<0.001$. 

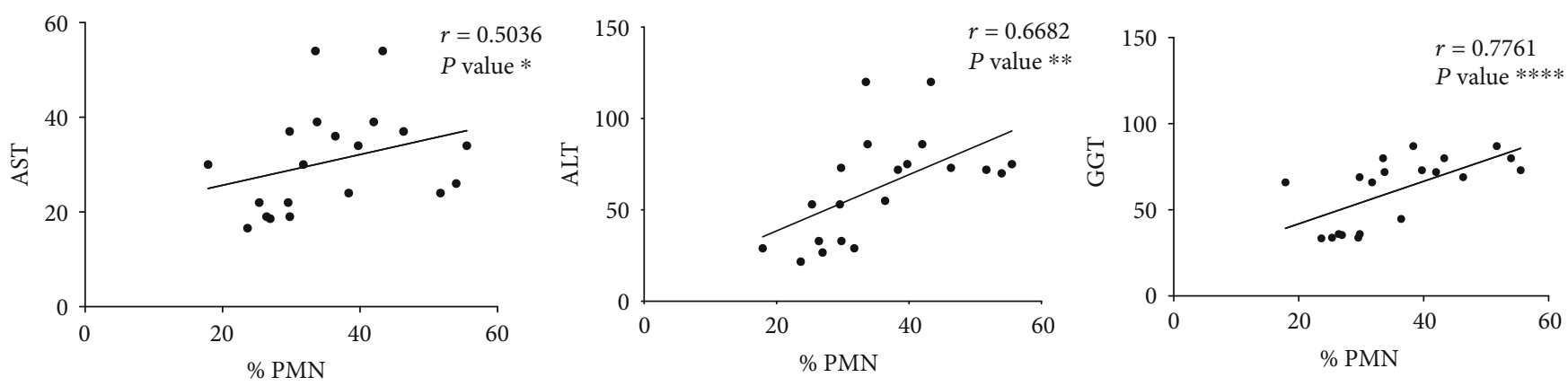

(a)

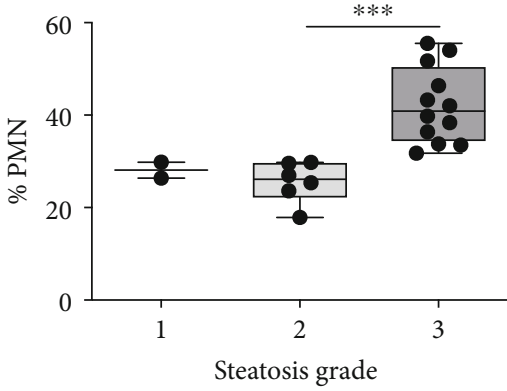

(b)

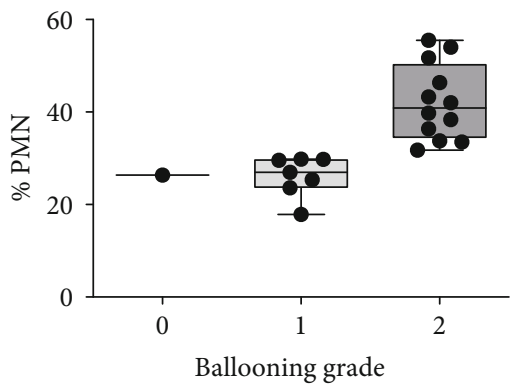

(d)

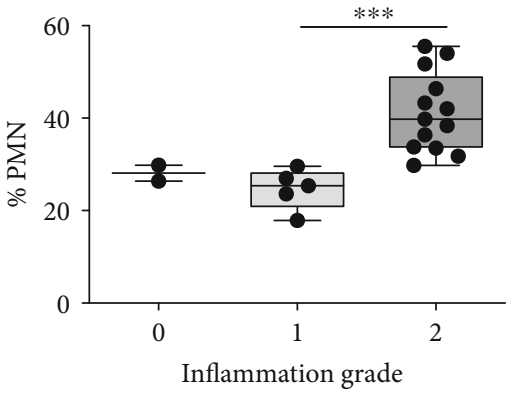

(c)

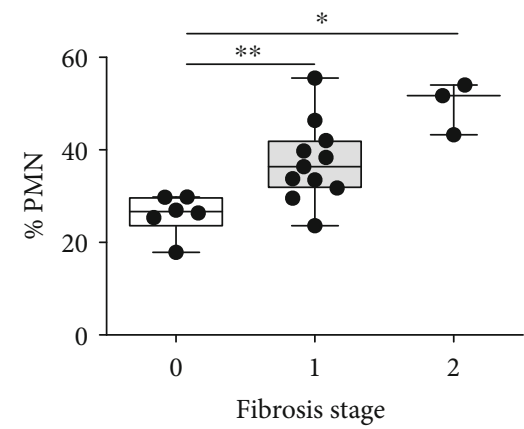

(e)

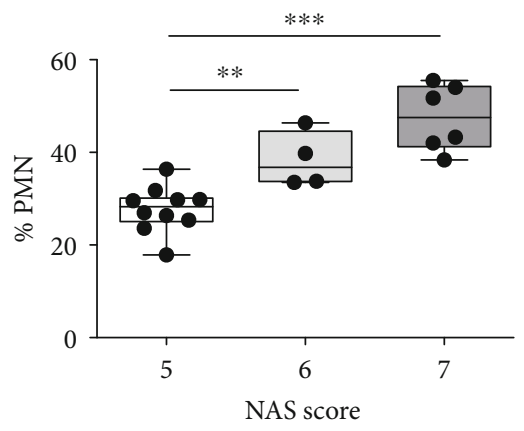

(f)

FIGURE 2: PMN frequency of NASH patients correlate with liver damage. (a) Spearman correlation ( $r$ ) between the frequency of freshly PMNs isolated from peripherical blood of NASH patients $(n=20)$ and liver enzymes aspartate aminotransferase (AST), alanine aminotransferase (ALT), and $\gamma$-glutamyl transferase (GGT) in serum $(r=0.536, r=6682$, and $r=7761$, respectively), data derived from medical records of patients. $P$ value, ${ }^{*} P<0.05,{ }^{* *} P<0.01,{ }^{* * *} P<0.001$, and ${ }^{* * * *} P<0.0001$. (b-f) Boxplots represent the association between frequency calculated by flow cytometry analysis as a percentage of $\mathrm{CD} 16^{\text {high }} \mathrm{CD} 62 \mathrm{~L}^{\text {high }} \mathrm{CD} 11 \mathrm{~b}^{\text {high }}$ of PMNs in NASH patients $(n=20)$ and histological severity of liver disease evaluated by steatosis grade (b), inflammation grade (c), hepatocellular ballooning (d), fibrosis stage (e), and NAS activity score (f). All data are represented as mean \pm SD. Each dot in the box-plot represents one subject/experiment $(n=20$ ). Each subject has been studied in an independent experiment. Plots are representative for three independent experiments with one sample per experiment. Mann-Whitney unpaired test, 2 -tailed. $P$ value, ${ }^{*} P<0.05,{ }^{* *} P<0.01,{ }^{* * *} P<0.001, * * * * P<0.0001$. 

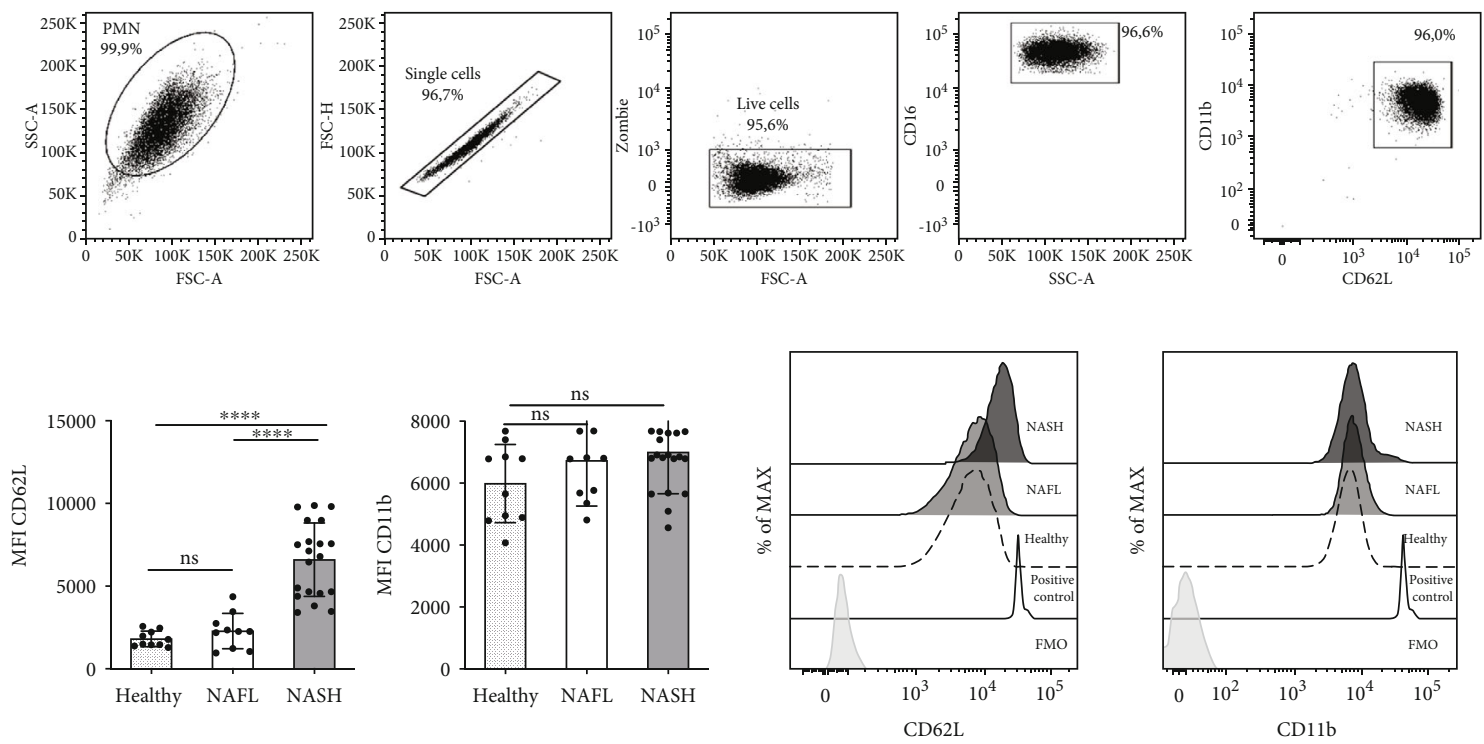

(a)
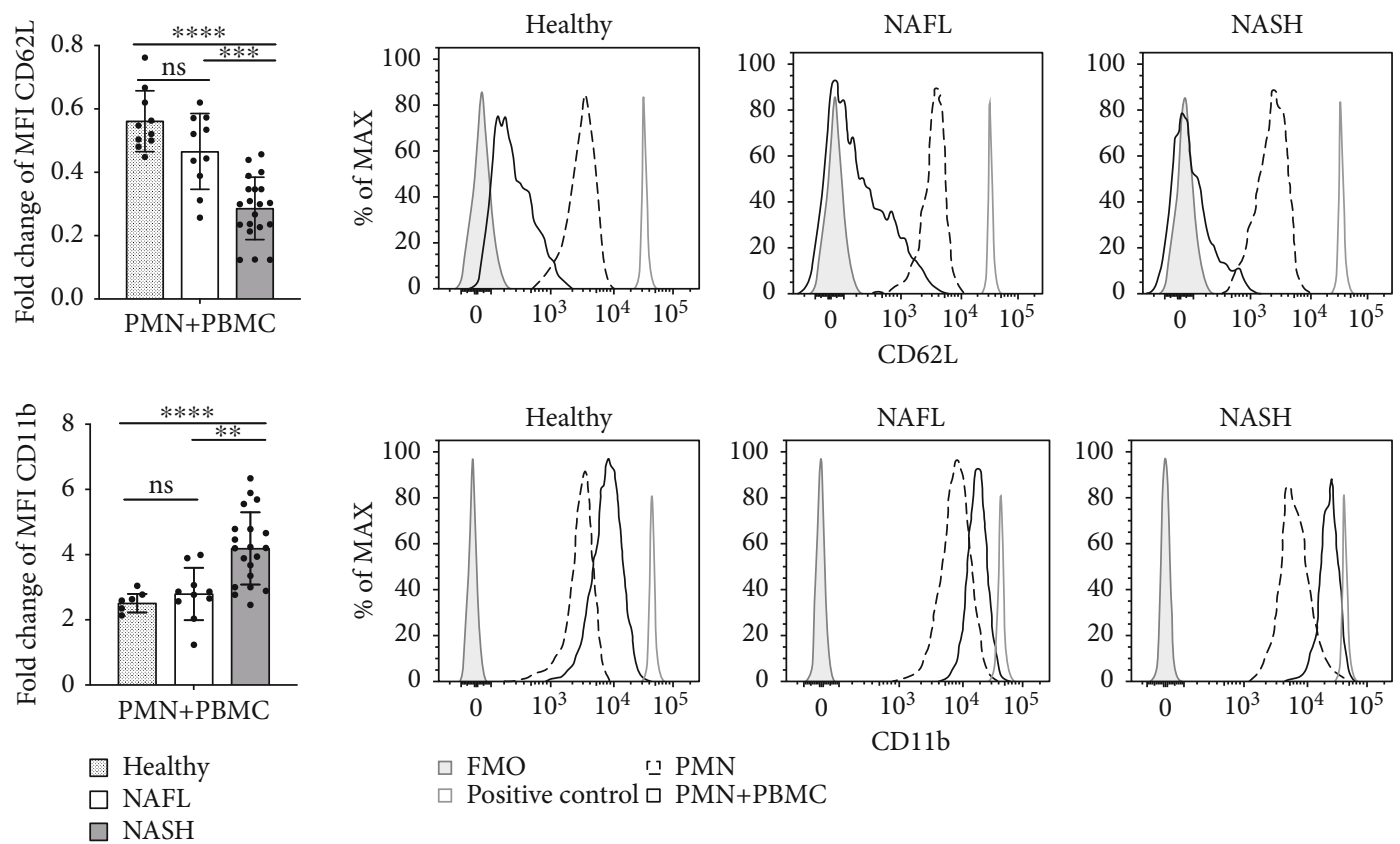

(b)

FIgURE 3: PMNs activated phenotype of NASH patients. (a) Top panels, representative flow cytometry gating strategy (NASH patients) for identification ex vivo PMNs. FACS plots displaying, SSC-A versus FSC-A, singlet cells (FSC-H versus FSC-A), live cells (Zombie versus FSCA), CD16 versus SSC-A, CD11b versus CD62L in ex vivo PMNs analysis. Bottom-left: histogram of mean fluorescence intensity (MFI) of CD62L and CD11b of ex vivo PMNs flow cytometric analysis obtained from healthy donors $(n=10)$, NAFL $(n=10)$, and NASH patients $(n=20)$. Bottom-right: representative histogram of flow cytometry analysis with an overlay showing CD62L and CD11b MFI in gated $\mathrm{CD} 16^{\mathrm{High}} \mathrm{CD} 62 \mathrm{~L}^{\mathrm{High}} \mathrm{CD} 11 \mathrm{~b}^{\mathrm{High}}$ of healthy donors (dashed line), NAFL (light gray), and NASH patients (dark gray). FMO control was represented by a gray histogram. Flow cytometry compensation beads positive control was represented by gray line. (b) PMNs obtained from healthy donors, NAFL, and NASH patients after $24 \mathrm{~h}$ of coculture with (PMNs+PBMCs) or without PBMCs (PMNs). Left panels: fold changes of CD62L (top) and CD11b (bottom) of MFI gated in CD16 ${ }^{\text {High }}$ CD62L ${ }^{\text {Dim }}$ CD11b ${ }^{\text {High }}$ in healthy donors $(n=10)$, NAFL $(n=10)$, and NASH patients $(n=20)$. Fold change was calculated comparing PMNs with PBMCs respect to PMNs without PBMCs. Right panels: representative histogram with an overlay showing CD62L (top) and CD11b (bottom) MFI in gated CD16 ${ }^{\mathrm{High}} \mathrm{CD} 62 \mathrm{~L}^{\mathrm{High}} \mathrm{CD} 11 \mathrm{~b}^{\mathrm{High}}$ after $24 \mathrm{~h}$ of coculture with PBMC in healthy donors, NAFL, and NASH patients. Histogram with dashed line represents PMN, PMNs+PBMCs are represented by a dark line. FMO control was represented by gray histogram and Lipopolysaccharide (LPS) positive control was represented by a gray line. In all histograms reported, for each subgroup, the distribution of each subject/experiment is shown, and results are expressed as mean \pm SD. Each dot represents one subject/experiment. Each subject has been studied in an independent experiment. In all experiments, statistics were performed by Mann-Whitney unpaired test, 2 -tailed. $P$ value, ${ }^{* *} P<0.01,{ }^{* * *} P<0.001$, ns: nonsignificant. 

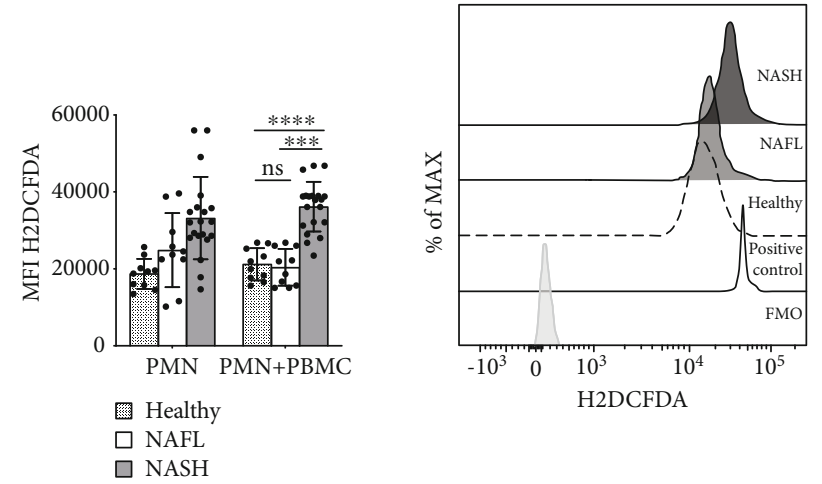

(a)
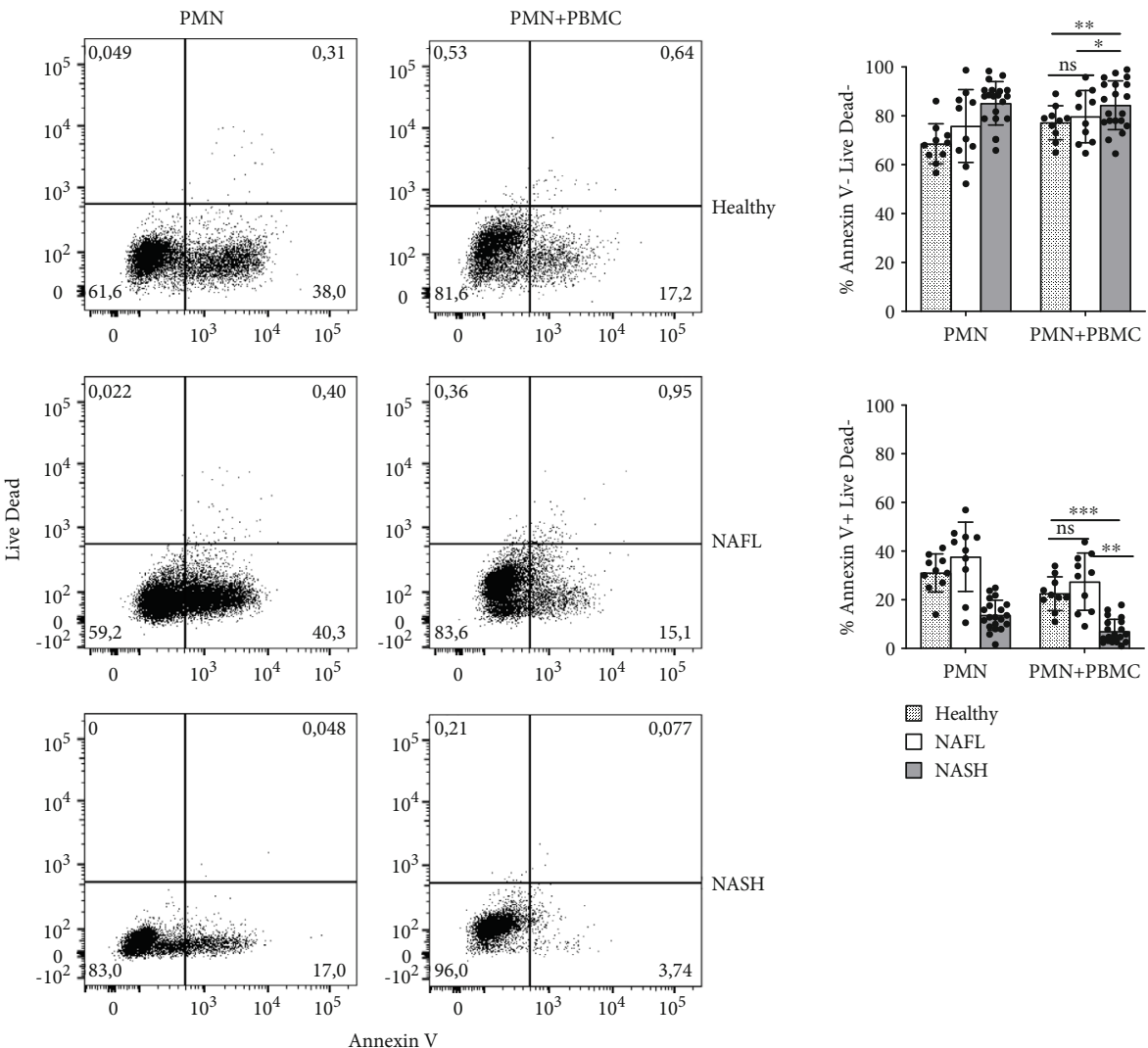

圈 Healthy

$\square$ NAFL

$\square \mathrm{NASH}$

(b)

FIgURE 4: PMN ROS intracellular production and viability. (a) PMNs obtained from healthy donors, NAFL, and NASH patients after $24 \mathrm{~h}$ of coculture, with (PMNs+PBMCs) or without PBMCs (PMNs), were stained with H2DCF-DA probe for intracellular production of ROS and (b) Annexin-V/Live Dead for vitality. (a) Left panel: histogram of H2DCFDA (ROS) MFI of CD16 ${ }^{\mathrm{High}} \mathrm{CD} 6 \mathrm{~L}^{\mathrm{High}} \mathrm{CD} 11 \mathrm{~b}^{\mathrm{High}}$ cell subset obtained from healthy donors $(n=10)$, NAFL $(n=10)$, and NASH $(n=20)$. Right panel: representative histogram of flow cytometry analysis with an overlay showing H2DCFDA (ROS) MFI in gated CD16 ${ }^{\mathrm{High}}$ CD62 $\mathrm{L}^{\mathrm{Dim}} \mathrm{CD} 11 \mathrm{~b}^{\mathrm{High}}$ of healthy donors (dashed line), NAFL (light gray), and NASH patients (dark gray), after $24 \mathrm{~h}$ of coculture with PBMCs. FMO control is represented by gray histogram and LPS positive control by a gray line. (b) Left panel: representative plots of PMNs cell death of healthy donors, NAFL, and NASH patients, after $24 \mathrm{~h}$, with (PMNS+PBMCs) or without PBMCs (PMNs). Right panel: percentage of live (Annexin-V, Live Dead negative) and early apoptotic (Annexin-V positive, Live Dead negative) of PMNs obtained from healthy donors $(n=10)$, NAFL $(n=10)$, and NASH patients $(n=20)$.In all histograms reported, for each subgroup, the distribution of each subject/experiment is showed and results are expressed as mean \pm SD. Each dot represents one subject/experiment. Each subject has been studied in an independent experiment. In all experiments, statistics were performed by Mann-Whitney unpaired test, 2 -tailed. $P$ value, ${ }^{*} P<0.05,{ }^{* *} P<0.01,{ }^{* * *} P<0.001,{ }^{* * * *} P<0.0001$, ns: nonsignificant. 
3.5. PMNs of NASH Patients Strongly Suppress $\mathrm{CD}^{+}$and $C D 8^{+} T$ Cells Proliferation and Activation. Since PMNs of NASH patients showed a more active phenotype, we looked at their capacity in affecting lymphocyte proliferation and activation. CFSE-based assay allowed us to assess the PMNs ability in modulating lymphocyte proliferation. PBMCs were isolated from healthy donors, NAFL, and NASH patients, labeled with proliferation tracer (CFSE) and stimulated with beads $\mathrm{aCD} 3 / \mathrm{aCD} 28$, in the presence or absence of autologous PMNs. A representative gate strategy of CFSE labeled $\mathrm{CD} 4^{+}$and $\mathrm{CD} 8^{+}$ $T$ cells in the presence or not of autologous PMNs was shown in Supplementary Figure S5. After the CD3/aCD28 stimulation, both $\mathrm{CD}^{+}$and $\mathrm{CD}^{+} \mathrm{T}$ cells enhanced their proliferation in healthy donors and patients. Interestingly, the PMNs/PBMC coculture induced a significant decrease of $\mathrm{CD}^{+}$and $\mathrm{CD} 8^{+} \mathrm{T}$ cell proliferation only in NASH patients (Figure 5(a)).

To determine whether PMNs were able to modulate the activation of $\mathrm{CD}^{+}$and $\mathrm{CD} 8^{+} \mathrm{T}$ cells, we looked at the expression of the $\mathrm{T}$ cell activation marker, CD25. We also evaluated the ability of $\mathrm{CD}^{+}$and $\mathrm{CD} 8^{+} \mathrm{T}$ cells to produce ROS. $\mathrm{CD}^{+}$and $\mathrm{CD} 8^{+} \mathrm{T}$ cells of NASH patients, after coculture with autologous PMNs, showed a significant decrease of CD25 expression levels respect to healthy donors and NAFL patients (Figure 5(b)). We observed a higher baseline ROS production CD4+ and CD8+ T cells of NASH patients compared to healthy donors and NAFL patients (Figure 5(c)). On the contrary, accordingly with the downregulation of CD25, ROS production was significantly decreased in $\mathrm{PMN}+\mathrm{PBMC}$ of NASH patients respect to healthy donors and NAFL patients (Figure 5(c)). This behavior was in line with the observed lack of apoptosis (Figure 5(d)).

\section{Discussion}

At the early stage, neutrophil infiltration in the liver represents one of the typical histological characteristics of NASH patients and animal models [37-39]. In response to chemotactic factors released by resident cells, $\mathrm{KC}$, and $\mathrm{T}$ cells, PMNs migrate from the bloodstream to the liver through the sinusoidal endothelial fenestrae. PMN infiltration contributes to the progression of tissue damage through the release of proinflammatory mediators, such as MPO and ROS [17, $18,37]$. These factors are involved in lipid peroxidation and hepatic stellate cell migration, facilitating cellular injury and fibrosis. The key role of neutrophils in the pathogenesis of NASH diseases was supported by demonstrating the increase of circulating levels and activity of neutrophils elastase during the curse of this pathology $[39,40]$. In NASH animal models, these proinflammatory cells regulate the ceramide metabolism, which is also involved in the pathogenesis of NASH [41].

Neutrophils show a strategic role not only in the early stage of NAFL/NASH disease but also in promoting the progression of NASH toward hepatocellular carcinoma (HCC) [42]. In particular, high levels of neutrophil extracellular traps (NETs) were found in the serum of patients with $\mathrm{NASH}$, these clinical observations have been confirmed in mice models in which NET formation contributes to the progression of HCC [42].
Interestingly, in the last years, it has become increasingly evident that PMNs are engaged in complex bidirectional interaction with $\mathrm{T}$ cells, establishing correct environmental conditions to initiate, amplify, and/or suppress adaptive immune effector responses [20, 21]. Thus, PMNs play an important role in the regulation of $\mathrm{T}$ cell immune response, worsening or improving the state of inflammation and the progression of diseases [20,37]. Nowadays, the crosstalk between PMNs and T lymphocytes, during NAFLD, has not been evaluated yet, but might have an important role in the progression of NAFL towards NASH. In fact, although it has been reported the highest number of PMNs in NASH patients $[38,43]$, an in-depth study of their complex interplay has not been already developed.

In our cohort of 30 NAFL/NASH patients, we observed a high Neutrophil-to-lymphocyte ratio (see Table 1) and a significant increase of PMNs frequency in the peripheral blood of NASH patients. In line with other studies [43], in the serum of our cohort of NASH patients, we observed an increase of IL-8, a neutrophil chemotactic factor. IL-8 might be involved in driving PMN mobilization towards the site of inflammation $[18,43]$. In addition, the increased levels of HMGB1, ARG1, and MPO indicate a higher activation of PMNs, leaving to hypothesize the main role of these cells in NASH progression. The data reported above were also confirmed by both a positive correlation between the frequency of PMNs and AST, ALT, and GGT parameters, and the association of neutrophils activity with the severity of liver disease (Figures 2(a) and 2(b)).

Expression changes in cell adhesion molecules, such as CD62L and CD11b, correlate with increased chemotaxis, transendothelial migration, and leukocyte activation [29]. Ex vivo PMNs deriving from healthy donors, NAFL, and NASH patients showed a resting phenotype characterized by CD16 ${ }^{\text {high }}$ CD62L ${ }^{\text {high }}$ and CD11b $b^{\text {high }}$ expression. Notably, we highlighted an overexpression of CD62L on the surface of PMNs of NASH patients respect to healthy donors and NAFL patients (Figure 3(a)). CD62L acts as a homing receptor and is required for the capture of circulating PMNs on activated endothelium [13, 14, 44-46]; thus, its overexpression could indicate a higher mobilization of PMNs towards the site of inflammation. No modulation was observed for CD11b in PMNs (Figure 3(a)). The lack of differences in the baseline expression levels of $\mathrm{CD} 11 \mathrm{~b}$ among all the three different groups of patients suggests a resting-state of circulating PMNs. Accordingly with literature, circulating PMNs of our cohort of patients seem to be not active because of their expression of low levels of CD11b that are associated with inactive conformation of ligand binding site of PMNs [47-49].

Interestingly, PMNs in coculture with autologous PBMCs showed an activated phenotype $\left(\mathrm{CD} 16^{\mathrm{High}} \mathrm{CD} 62 \mathrm{~L}^{\text {Dim }}\right.$ $\left.\mathrm{CD}_{1} 1 \mathrm{~b}^{\mathrm{High}}\right)$. Notably, PMNs from NASH patients, conversely respect to healthy donors and NAFL, when cocultured with PBMCs, acquired a strong active phenotype as revealed by the shedding of $\mathrm{CD} 62 \mathrm{~L}$, the upregulation of $\mathrm{CD} 11 \mathrm{~b}$ (Figure 3(b)) and the increased intracellular production of ROS [50] (Figure 4(a)). These evidences supported the idea that PMNs of NASH patients have an intrinsic capacity to be activated in the presence of PBMCs. 

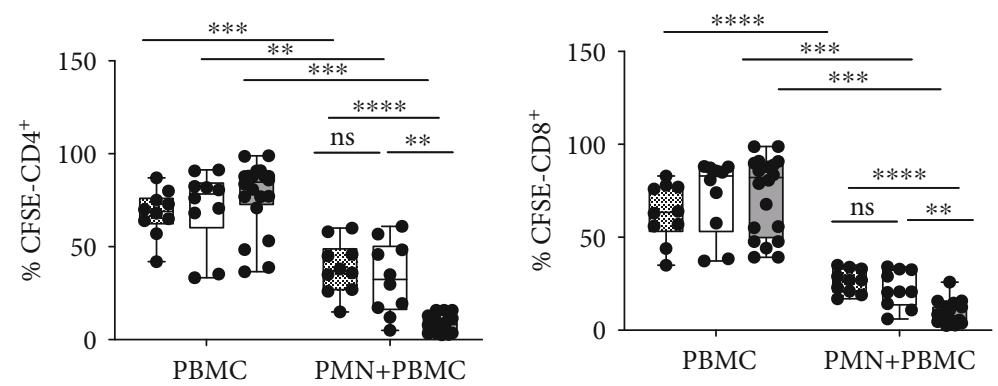
Healthy
$\square$ NAFL
$\square \mathrm{NASH}$

(a)
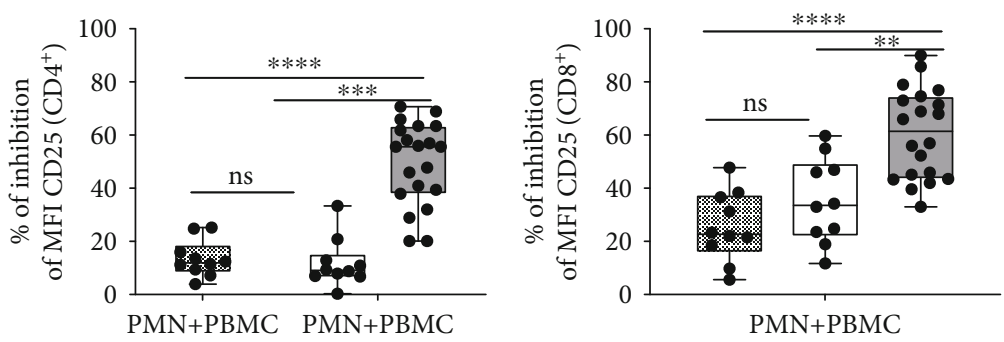
Healthy
$\square$ NAFL
$\square \mathrm{NASH}$

(b)

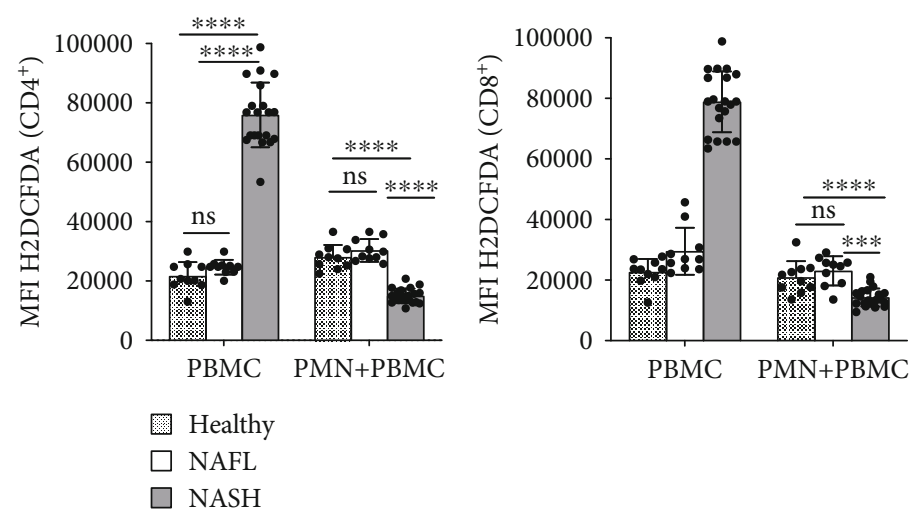

(c)

Figure 5: Continued. 


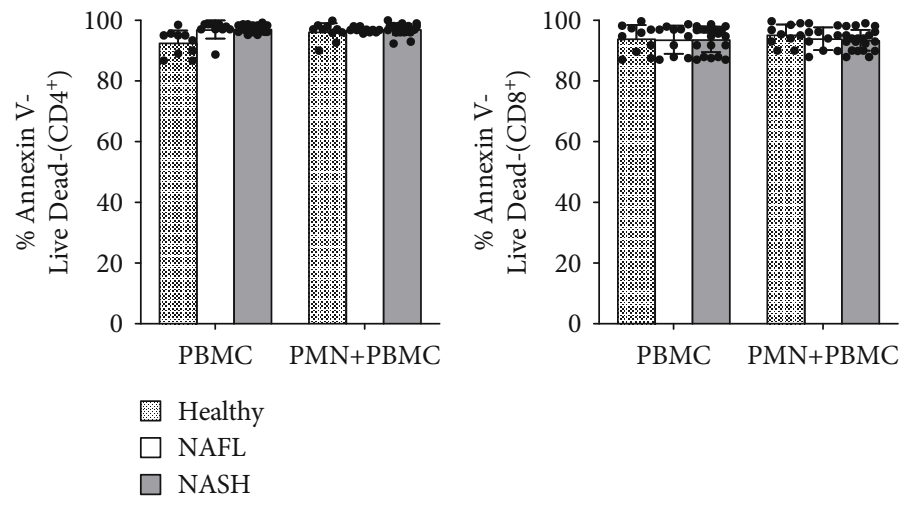

(d)

Figure 5: Proliferation and activation of $\mathrm{CD}^{+}$and $\mathrm{CD}^{+} \mathrm{T}$ cells cocultured with PMNs. (a-d) In all experiments, PBMCs obtained from healthy donors, NAFL, and NASH patients were stimulated with aCD3/aCD28 and cultured in the presence or in absence of autologous PMNs for $96 \mathrm{~h}$. The capacity of PMN to suppress T cells was evaluated by measuring proliferating T cells (CFSE dilution). (a) Top panels: frequency calculated by flow cytometry analysis as the percentage of $\mathrm{CD}^{+}$(left) and $\mathrm{CD}^{+}$(right) $\mathrm{T}$ cell proliferation is measured in healthy donors $(n=10)$, NAFL $(n=10)$, and NASH $(n=20)$ by CFSE assay. Each dot in the graph represents a single subject. (b) The expression of activation marker (CD25) on T cells was analyzed by flow cytometry, after $96 \mathrm{~h}$ of cultured with PMNs. Dot plots show the percentage of inhibition of CD25 MFI [(MFI increase of CD25 of PBMC - MFI increase of CD25 of PBMC + PMN $) /($ MFI increase of CD 25 of PBMC) x 100\%] on CD4 ${ }^{+}$(left) and $\mathrm{CD}^{+}$(right) T cells in healthy donors $(n=10)$, NAFL $(n=10)$, and NASH $(n=20)$. Each dot in the dot-plot ( $\mathrm{a}$ and $\mathrm{b}$ ) represents one subject/experiment. Each subject has been studied in an independent experiment. Plots are representative for two independent experiment with one sample per experiment. (c-d) After $96 \mathrm{~h}$ of coculture with PMNs, T cells were stained with H2DCF-DA probe for identifying intracellular production of ROS, and Annexin-V/Live Dead for their vitality. (c) Histograms show the percentage of H2DCFDA (ROS) MFI on gated CD4 ${ }^{+}$(left) and CD8 ${ }^{+}$(right) T cells in healthy donors $(n=10)$, NAFL $(n=10)$, and NASH $(n=20)$. (d) Frequency of cell death CD4 ${ }^{+}$(left) and CD8 ${ }^{+}$(right) T cells in healthy donors $(n=10)$, NAFL $(n=10)$, and NASH $(n=20)$. Survival is defined as the percentage of Annexin V, Live Dead double negative cells. In all histograms reported (c and d), for each subgroup the distribution of each subject/experiment is showed and results are expressed as mean \pm SD. Each dot represents one subject/experiment. Each subject has been studied in an independent experiment. In all experiments (a-d), statistics were performed by Mann-Whitney unpaired test, 2 -tailed. $P$ value, ${ }^{*} P<0.05,{ }^{* *} P<0.01, * * * P<0.001, * * * * P<0.0001$, ns: nonsignificant.

Moreover, PMNs of our cohort of NASH patients, in the presence of PBMCs, have longer survival and less apoptosis survival compared to PMNs of healthy donors and NAFL patients (Figure 4(b)). All these observations suggest that PMNs of NASH patients, in the presence of PBMCs, can contribute in worsening liver inflammatory status. The apoptosis observed in PMNs of healthy donors and NAFL patients could be a determinant for the effective resolution of liver inflammation.

Finally, for the first time, we have demonstrated that PMNs of NASH patients were able to suppress the proliferation and the activation of autologous $\mathrm{CD}^{+}$and $\mathrm{CD}^{+} \mathrm{T}$ cells compared to PMNs from healthy donors and NAFL patients (Figure 5(a)). This behavior is highlighted by the downregulation in $\mathrm{CD}^{+}$and $\mathrm{CD}^{+} \mathrm{T}$ cells of the expression of CD25 (Figure 5(b)) together with the low intracellular concentration of ROS (Figure 5(c)) and lack of apoptosis (Figure 5(d)).

\section{Conclusion}

Taking all into consideration, our data revealed an extremely different behavior and function of PMNs in NASH respect to NAFL patients and healthy donors. These findings highlight that, in the presence of steatohepatitis, an immunological tolerance might take place; thus contributing to the progression of liver disease. Furthermore, a strong suppression of
$\mathrm{CD}^{+}$and $\mathrm{CD}^{+} \mathrm{T}$ cell proliferation and activation may induce, over time, an inadequate immune-surveillance of liver damage, making patients more susceptible to liver damage progression.

The modulation of PMNs activity, in NASH patients, might represent a novel and effective therapeutic approach to counteract fat-related liver damage progression. Thus, the use of molecules able to modulate PMNs activity could redirect the immunosuppressive properties of PMNs and boost T-cell responses in NASH patients.

\section{Abbreviations}

NAFLD: $\quad$ Nonalcoholic fatty liver disease

NAFL: $\quad$ Nonalcoholic fatty liver

NASH: Nonalcoholic steatohepatitis

PMNs: $\quad$ Polymorphonuclear leukocytes

MPO: $\quad$ Myeloperoxidase

ROS: $\quad$ Reactive oxygen species

ARG1: $\quad$ Arginase 1

PBMCs: $\quad$ Peripheral blood mononuclear cells

CFSE: Carboxyfluoresceindiacetate, succinimidyl ester

H2DCF-DA, 2' $: 7^{\prime}$-dichlorodihydrofluorescein diacetate

MFI: $\quad$ Mean fluorescence intensity

PB: $\quad$ Peripheral blood

LPS: $\quad$ Lypopolysaccharide. 


\section{Data Availability}

The data used to support the findings of this study are included within the article.

\section{Conflicts of Interest}

The research was conducted in the absence of any commercial or financial relationships that could be construed as a potential conflict of interest.

\section{Authors' Contributions}

All authors have been involved in the discussion and the drafting of the article and have approved the final version for publication. They have full access to all data, taking responsibility for their integrity and analysis accuracy. LA and CP performed the experiments; LA, CP, ET, SJS, and $\mathrm{CB}$ analyzed the results and $\mathrm{CB}$ and GI enrolled patients. LA, CP, ET, and CB provided important contributions to the conception of the work as well as to the interpretation of the data and manuscript writing. Laura Antonucci and Cristiana Porcu contributed equally to this work.

\section{Acknowledgments}

This work was supported by a grant of the Fondazione Francesco Balsano, the "Fondo per gli Investimenti della Ricerca di Base (FIRB)" (Prot. RBAP10TPXK CUP: B11J10000300008) and by the Gilead Felloship Program.

\section{Supplementary Materials}

Supplementary Figure S1: representative flow cytometry gating strategy of PMNs of NASH patients after $24 \mathrm{hrs}$ of coculture with PBMC. FACS plots displaying, CD16 versus SSC-A, CD11b versus CD62L in coculture PMN analysis. Supplementary Figure S2: fold change in the ROS production of PMNs and PMNs coculture with PBMCs. All data are represented as mean \pm standard deviation $\left({ }^{*} P<0.05\right)$. Supplementary Figure S3: representative gating strategy of PMNs derived from NASH patients cocultured or not with PBMCs. The neutrophil population (PMNs) in peripheral blood was distinguished by flow cytometry based on their forward(FSC-A) and side-scatter (SSC-A) characteristics. (A) PBMCs cultured without $\mathrm{CD} 3 / \mathrm{CD} 28$ stimulation have been used as internal control. PBMCs obtained from NASH patients were stimulated with aCD3/aCD28 and cultured with (B) and without (C) autologous PMNs for 96h. Capacity of PMNs to suppress $\mathrm{T}$ cells was evaluated by measuring proliferating $\mathrm{T}$ cells (CFSE dilution). (Supplementary Materials)

\section{References}

[1] M. Noureddin and A. J. Sanyal, "Pathogenesis of NASH: the Impact of Multiple Pathways," Current Hepatology Reports, vol. 17, no. 4, pp. 350-360, 2018.

[2] Z. Younossi, Q. M. Anstee, M. Marietti et al., "Global burden of NAFLD and NASH: trends, predictions, risk factors and prevention," Nature Reviews Gastroenterology \& Hepatology, vol. 15, no. 1, pp. 11-20, 2018.

[3] N. Tanaka, T. Kimura, N. Fujimori, T. Nagaya, M. Komatsu, and E. Tanaka, "Current status, problems, and perspectives of non-alcoholic fatty liver disease research," Journal of Gastroenterology, vol. 25, no. 2, pp. 163-177, 2019.

[4] G. Cholankeril, R. Patel, S. Khurana, and S. K. Satapathy, "Hepatocellular carcinoma in non-alcoholic steatohepatitis: current knowledge and implications for management," World Journal of Hepatology, vol. 9, no. 11, pp. 533-543, 2017.

[5] N. Alkhouri and A. Scott, "An update on the pharmacological treatment of nonalcoholic fatty liver disease: beyond lifestyle modifications," Clinical Liver Disease, vol. 11, no. 4, pp. 8286, 2018.

[6] V. W.-S. Wong and A. K. Singal, "Emerging medical therapies for non-alcoholic fatty liver disease and for alcoholic hepatitis," Translational Gastroenterology and Hepatology, vol. 4, p. 53, 2019.

[7] J. A. Del Campo, P. Gallego, and L. Grande, "Role of inflammatory response in liver diseases: Therapeutic strategies," World Journal of Hepatology, vol. 10, no. 1, pp. 1-7, 2018.

[8] M. Arrese, D. Cabrera, A. M. Kalergis, and A. E. Feldstein, "Innate immunity and inflammation in NAFLD/NASH," Digestive Diseases and Sciences, vol. 61, no. 5, pp. 1294-1303, 2016.

[9] P. Huebener, J.-P. Pradere, C. Hernandez et al., "The HMGB1/RAGE axis triggers neutrophil-mediated injury amplification following necrosis," Journal of Clinical Investigation, vol. 125, no. 2, pp. 539-550, 2015.

[10] H. Bi, Y. Zhang, S. Wang et al., "Interleukin-8 promotes cell migration via CXCR1 and CXCR2 in liver cancer," Oncology Letters, vol. 18, no. 4, 2019.

[11] S. K. Raghuwanshi, Y. Su, V. Singh, K. Haynes, A. Richmond, and R. M. Richardson, "The chemokine receptors CXCR1 and CXCR2 couple to distinct G protein-coupled receptor kinases to mediate and regulate leukocyte functions," Journal of Immunology, vol. 189, no. 6, pp. 2824-2832, 2012.

[12] R. Xu, H. Huang, Z. Zhang, and F. S. Wang, "The role of neutrophils in the development of liver diseases," Cellular \& Molecular Immunology, vol. 11, no. 3, pp. 224-231, 2014.

[13] C. Rosales, "Neutrophil: a cell with many roles in inflammation or several cell types?," Frontiers in Physiology, vol. 9, 2018.

[14] K. Ley, C. Laudanna, M. I. Cybulsky, and S. Nourshargh, "Getting to the site of inflammation: the leukocyte adhesion cascade updated," Nature Reviews Immunology, vol. 7, no. 9, pp. 678-689, 2007.

[15] A. Ivetic, H. L. Hoskins Green, and S. J. Hart, "L-selectin: A Major Regulator of Leukocyte Adhesion, Migration and Signaling," Frontiers in Immunology, vol. 10, 2019.

[16] D. Begandt, S. Thome, M. Sperandio, and B. Walzog, "How neutrophils resist shear stress at blood vessel walls: molecular mechanisms, subcellular structures, and cell-cell interactions," Journal of Leukocyte Biology, vol. 102, no. 3, pp. 699-709, 2017.

[17] S. S. Rensen, V. Bieghs, S. Xanthoulea et al., "Neutrophilderived myeloperoxidase aggravates non-alcoholic steatohepatitis in low-density lipoprotein receptor-deficient mice," PLoS One, vol. 7, no. 12, p. e52411, 2012.

[18] M. A. Cassatella, N. K. Östberg, N. Tamassia, and O. Soehnlein, "Biological roles of neutrophil-derived granule 
proteins and cytokines," Trends in Immunology, vol. 40, no. 7, pp. 648-664, 2019.

[19] N. Magee, A. Zou, and Y. Zhang, "Pathogenesis of nonalcoholic steatohepatitis: interactions between liver parenchymal and nonparenchymal cells," BioMed Research International, vol. 2016, 11 pages, 2016.

[20] S. Costa, D. Bevilacqua, M. A. Cassatella, and P. Scapini, "Recent advances on the crosstalk between neutrophils and B or T lymphocytes," Immunology, vol. 156, no. 1, pp. 23-32, 2019.

[21] P. Scapini, O. Marini, C. Tecchio, and M. A. Cassatella, "Human neutrophils in the saga of cellular heterogeneity: insights and open questions," Immunological Reviews, vol. 273, no. 1, pp. 48-60, 2016.

[22] N. L. Bowers, E. S. Helton, R. P. H. Huijbregts, P. A. Goepfert, S. L. Heath, and Z. Hel, "Immune suppression by neutrophils in HIV-1 infection: role of PD-L1/PD-1 pathway," PLoS Pathogens, vol. 10, no. 3, p. e1003993, 2014.

[23] C. J. Darcy, G. Minigo, K. A. Piera et al., "Neutrophils with myeloid derived suppressor function deplete arginine and constrain T cell function in septic shock patients," Critical Care, vol. 18, no. 4, p. R163, 2014.

[24] K. Moses and S. Brandau, "Human neutrophils: their role in cancer and relation to myeloid-derived suppressor cells," Seminars in Immunology, vol. 28, no. 2, pp. 187-196, 2016.

[25] S. Singhal, P. S. Bhojnagarwala, S. O'Brien et al., "Origin and Role of a Subset of Tumor-Associated Neutrophils with Antigen- Presenting Cell Features in Early-Stage Human Lung Cancer," Cancer Cell, vol. 30, no. 1, pp. 120-135, 2016.

[26] E. B. Eruslanov, P. S. Bhojnagarwala, J. G. Quatromoni et al., "Tumor-associated neutrophils stimulate $\mathrm{T}$ cell responses in early-stage human lung cancer," The Journal of Clinical Investigation, vol. 124, no. 12, pp. 5466-5480, 2014.

[27] I. Mishalian, R. Bayuh, L. Levy, L. Zolotarov, J. Michaeli, and Z. G. Fridlender, "Tumor-associated neutrophils (TAN) develop pro-tumorigenic properties during tumor progression," Immunotherapy, vol. 62, no. 11, pp. 1745-1756, 2013.

[28] P. H. C. Leliefeld, L. Koenderman, and J. Pillay, "How Neutrophils Shape Adaptive Immune Responses," Frontiers in Immunology, vol. 6, 2015.

[29] I. Mitroulis, V. I. Alexaki, I. Kourtzelis, A. Ziogas, G. Hajishengallis, and T. Chavakis, "Leukocyte integrins: role in leukocyte recruitment and as therapeutic targets in inflammatory disease," Pharmacology \& Therapeutics, vol. 147, pp. 123-135, 2015.

[30] S. Leoni, F. Tovoli, L. Napoli, I. Serio, S. Ferri, and L. Bolondi, "Current guidelines for the management of non-alcoholic fatty liver disease: a systematic review with comparative analysis," World Journal of Gastroenterology, vol. 24, no. 30, pp. 33613373, 2018.

[31] M. Matsuda and R. A. DeFronzo, "Insulin sensitivity indices obtained from oral glucose tolerance testing: comparison with the euglycemic insulin clamp," Diabetes Care, vol. 22, no. 9, pp. 1462-1470, 1999.

[32] R. B. Merriman and T. T. Tran, "AASLD practice guidelines: the past, the present, and the future," Hepatology, vol. 63, no. 1, pp. 31-34, 2016.

[33] S. H. Saverymuttu, A. E. Joseph, and J. D. Maxwell, "Ultrasound scanning in the detection of hepatic fibrosis and steatosis," British Medical Journal (Clinical Research Ed.), vol. 292, no. 6512, pp. 13-15, 1986.
[34] N. T. Gunn and M. L. Shiffman, "The use of liver biopsy in nonalcoholic fatty liver disease: when to biopsy and in whom," Clinics in Liver Disease, vol. 22, no. 1, pp. 109119, 2018.

[35] D. E. Kleiner, E. M. Brunt, M. van Natta et al., "Design and validation of a histological scoring system for nonalcoholic fatty liver disease," Hepatology, vol. 41, no. 6, pp. 13131321, 2005.

[36] A. Cossarizza, H. D. Chang, A. Radbruch et al., "Guidelines for the use of flow cytometry and cell sorting in immunological studies," European Journal of Immunology, vol. 47, no. 10, pp. 1584-1797, 2017.

[37] M. E. Inzaugarat, N. E. Ferreyra Solari, L. A. Billordo, R. Abecasis, A. C. Gadano, and A. C. Cherñavsky, "Altered phenotype and functionality of circulating immune cells characterize adult patients with nonalcoholic steatohepatitis," Journal of Clinical Immunology, vol. 31, no. 6, pp. 1120-1130, 2011.

[38] V. Papayannopoulos, "Neutrophil extracellular traps in immunity and disease," Nature Reviews Immunology, vol. 18, no. 2, pp. 134-147, 2018.

[39] S. Zang, L. Wang, X. Ma et al., "Neutrophils play a crucial role in the early stage of nonalcoholic steatohepatitis via neutrophil elastase in mice," Cell Biochemistry and Biophysics, vol. 73, no. 2, pp. 479-487, 2015.

[40] A.-M. Mirea, E. J. M. Toonen, I. van den Munckhof et al., "Increased proteinase 3 and neutrophil elastase plasma concentrations are associated with non-alcoholic fatty liver disease (NAFLD) and type 2 diabetes," Molecular Medicine, vol. 25, no. 1, p. 16, 2019.

[41] J. Chen, B. Liang, D. Bian et al., "Knockout of neutrophil elastase protects against western diet induced nonalcoholic steatohepatitis in mice by regulating hepatic ceramides metabolism," Biochemical and Biophysical Research Communications, vol. 518, no. 4, pp. 691-697, 2019.

[42] D. J. van der Windt, V. Sud, H. Zhang et al., "Neutrophil extracellular traps promote inflammation and development of hepatocellular carcinoma in nonalcoholic steatohepatitis," Hepatology, vol. 68, no. 4, pp. 1347-1360, 2018.

[43] I. H. Bahcecioglu, M. Yalniz, H. Ataseven et al., "Levels of serum hyaluronic acid, TNF-alpha and IL-8 in patients with nonalcoholic steatohepatitis," Hepato gastroenterology, vol. 52, no. 65, pp. 1549-1553, 2005.

[44] N. Strydom and S. M. Rankin, "Regulation of circulating neutrophil numbers under homeostasis and in disease," Journal of Innate Immunity, vol. 5, no. 4, pp. 304-314, 2013.

[45] P. X. Liew and P. Kubes, "The Neutrophil's Role During Health and Disease," Physiological Reviews, vol. 99, no. 2, pp. 1223-1248, 2019.

[46] A. Ivetic, "A head-to-tail view of L-selectin and its impact on neutrophil behaviour," Cell and Tissue Research, vol. 371, no. 3, pp. 437-453, 2018.

[47] C. Feng, L. Zhang, L. Almulki et al., "Endogenous PMN sialidase activity exposes activation epitope on CD11b/CD18 which enhances its binding interaction with ICAM-1," Journal of Leukocyte Biology, vol. 90, no. 2, pp. 313-321, 2011.

[48] C. L. Abram and C. A. Lowell, "The ins and outs of leukocyte integrin signaling," Annual Review of Immunology, vol. 27, no. 1, pp. 339-362, 2009. 
[49] A. McDowall, B. Leitinger, P. Stanley, P. A. Bates, A. M. Randi, and N. Hogg, "The I Domain of Integrin Leukocyte Functionassociated Antigen-1 Is Involved in a Conformational Change Leading to High Affinity Binding to Ligand Intercellular Adhesion Molecule 1 (ICAM-1)," Journal of Biological Chemistry, vol. 273, no. 42, pp. 27396-27403, 1998.

[50] J. Pillay, V. M. Kamp, E. van Hoffen et al., “A subset of neutrophils in human systemic inflammation inhibits $\mathrm{T}$ cell responses through Mac-1," The Journal of Clinical Investigation, vol. 122, no. 1, pp. 327-336, 2012. 